C. Freiling, Department of Mathematics, California State University, San

Bernardino, California, 92407-2397 .

B. S. Thomson` Department of Mathematics, Simon Fraser University, B.C., Canada V5A 1S6.

\title{
SCATTERED SETS AND GAUGES
}

\begin{abstract}
An elementary and natural method for demonstrating that certain exceptional sets are scattered is presented.
\end{abstract}

In this note we wish to present a simple technique that can be used to establish that certain exceptional sets are scattered.

Recall that a set of real numbers is scattered if every nonempty subset has an isolated point. One sided versions have been considered in the past: a set is right [left] scattered if every nonempty subset has a point isolated on the right [left]; any such set is called semi-scattered. A set is splattered if every nonempty subset has a point isolated on one side at least. A splattered set may be expressed as the union of a right scattered set and a left scattered set. Scattered sets may, similarly, be viewed as the intersection of a right scattered set and a left scattered set.

The first explicit use of such ideas is in Cantor [1] where he uses the term separierte Mengen for a set that contains no subset dense-in-itself. In the first decades of this century, G. C. Young and W. H. Young made considerable use of scattered sets including left and right versions but employed no terminology. Denjoy introduced the term clairsemé into French language accounts and Hausdorff employed zerstreute Mengen in his writing. Hobson [7] in his account of the period used the notions but failed to employ any terminology (even avoiding the French term which, by then, was well known). Viola [10] (a student of Denjoy) is perhaps the first to study extensively the one sided

\footnotetext{
Key Words: scattered set

Mathematical Reviews subject classification: Primary: 26A21, 26A24

Received by the editors September 8,1994

* Research supported in part by a grant from NSF.

${ }^{\dagger}$ Research supported in part by a grant from NSERC.
} 
versions, under the terminology clairsemé à droit/gauche. We do not know when the English language term scattered first appeared. The language semiscattered as well as the new notion of splattered were introduced in [5]; in addition that article contains a number of games characterizing all of these concepts.

We show that every gauge function (that is a map $\delta: \mathbb{R} \rightarrow \mathbb{R}^{+}$) is naturally associated with a pair of left and right scattered sets and that these sets frequently play a key role in the classification of exceptional sets. The companion article [6] that appears also in this issue of the Exchange explores the scattered sets within a broader context of chains of open sets giving a better sense of the exact structure of these sets. For most purposes and applications, however, the reader will find that the following proposition suffices.

Proposition 1 Let $\delta$ be a gauge defined on all of $\mathbb{R}$ except possibly for some countable set. Then, except for a right [left] scattered set, every point $x$ is the limit from the right [left] of some sequence $\left\{x_{i}\right\}$ for which $\delta\left(x_{i}\right)$ is bounded above zero.

Proof. We suppose that $\delta(x)$ is defined and positive at every point excepting for $x$ in a countable set $N=\left\{z_{1}, z_{2}, z_{3}, \ldots\right\}$. Let $S_{r}=\left\{x: \lim _{y \rightarrow x+} \delta(y)=0\right\}$. We show that $S_{r}$ is right scattered. Let $A$ be an arbitrary nonempty subset of $S_{r}$. Choose $x_{1} \in A$. If $x_{1}$ is isolated from $A$ on the right, then we are done. Otherwise choose $y_{1}>x_{1}$ so that $z_{1} \notin\left(x_{1}, y_{1}\right)$ and so that, for all $x \in\left(x_{1}, y_{1}\right) \backslash N, \delta(x)<1$. Suppose that $\left(x_{n}, y_{n}\right)$ has been chosen so that $x_{n} \in A$ with $\left(x_{n}, y_{n}\right) \cap\left\{z_{1}, z_{2}, \ldots, z_{n}\right\}=\emptyset$ and so that, for all $x \in\left(x_{n}, y_{n}\right) \backslash N$, $\delta(x)<n^{-1}$. If $x_{n}$ is isolated from $A$ on the right, then we are done. Otherwise we can continue choosing $x_{n+1} \in\left(x_{n}, y_{n}\right) \cap A$ and then $y_{n+1} \in\left(x_{n+1}, y_{n}\right)$ such that

$$
\left(x_{n+1}, y_{n+1}\right) \cap\left\{z_{1}, z_{2}, \ldots, z_{n+1}\right\}=\emptyset
$$

and, for all $x$ in $\left(x_{n+1}, y_{n+1}\right) \backslash N, \delta(x)<(n+1)^{-1}$. This process cannot continue forever since, otherwise, there is a point $z_{0}$ in $\bigcap_{i=1}^{\infty}\left(x_{i}, y_{i}\right)$ so that $z_{0} \notin N$ and hence $\delta\left(z_{0}\right)<i^{-1}$ for all $i$ which is impossible.

Davies and Galvin [3] have characterized scattered sets of real numbers as countable $\mathcal{G}_{\delta}$-sets. This characterization can clarify Proposition 1 further. From the elementary theory of cluster sets one knows that the set

$$
S=\left\{x: \lim _{y \rightarrow x} \delta(y)=0\right\}
$$

must be countable for any gauge $\delta$. But then writing

$$
S=\bigcap_{n=1}^{\infty}\{x: \exists \epsilon>0,0<|y-x|<\epsilon \Rightarrow \delta(y)<1 / n\}
$$


exhibits this set as of type $\mathcal{G}_{\delta}$. This gives a different perspective on the gauge proposition.

To see this interplay between gauges and scattered sets let us show how Proposition 1 can be used to give an elementary proof of the fact that countable $\mathcal{G}_{\delta}$-sets of reals are scattered. (Topologists know that this holds in any Baire space.)

Proposition 2 A countable $\mathcal{G}_{\delta}$-set of real numbers is scattered.

Proof. Let $S$ be a countable $\mathcal{G}_{\delta}$-set. Then $S=\bigcap_{i=1}^{\infty} G_{i}$ where $\left\{G_{i}\right\}$ is a sequence of open sets, which we may assume to be decreasing. Define the gauge $\delta(x)=\max \left\{1 / n: x \notin G_{n}\right\}$ at each point not in the countable set $S$. Proposition 1 implies that

$$
\lim _{x \rightarrow s, x \notin S} \delta(x)=0
$$

only for $s$ in a scattered set. But each $s \in S$ has this property and so $S$ itself is scattered as required.

The remainder of this article is devoted to further applications of Proposition 1 showing how it provides a basic tool for establishing that many exceptional sets are scattered or semi-scattered. Our first application is to a characterization from Viola [10] of semiscattered sets.

Theorem 3 (Viola) Let $\mathcal{I}$ be a collection of open intervals with the property that for each $x \in \mathbb{R}$, with perhaps countably many exceptions, $x$ belongs to finitely many of the elements of $\mathcal{I}$. Then the set of right endpoints of the intervals of $\mathcal{I}$ is left scattered and set of left endpoints of the intervals of $\mathcal{I}$ is right scattered.

Proof. Let $N$ be the countable set of exceptions in the statement of the theorem. Define the gauge

$$
\delta(x)=\min \{1, x-a: x \in(a, b),(a, b) \in \mathcal{I}\}
$$

for each point $x$ not in $N$. Let $c$ be the left endpoint of an interval $(c, d) \in \mathcal{I}$. Then $\lim _{x \rightarrow c+, x \notin N} \delta(x)=0$. Hence, by Proposition 1, the set of such left endpoints is right scattered as required.

Our second example is a simple semiscattered property of real functions. This may be found in various forms elsewhere. For example, if we let $g$ denote the oscillation function of $f$, then it includes Viola's [10] observation that a function $f$ with a left hand derivative must be continuous off a right scattered set; Charzyński [2] employs a similar argument in a different context. 
Lemma 4 Let $g$ be a nonnegative function and $N$ a countable set. Suppose that $\lim \sup _{t \rightarrow 0+} t^{-1} g(x-t)<\infty$ for every $x \in \mathbb{R} \backslash N$. Then $\{x: g(x)>0\}$ is right scattered.

Proof. For each $x \notin N$ there is a $\delta>0$ and an $M$ such that $g(x-t)<M t$ for all $0<t<\delta$. By shrinking $\delta$ if necessary we can obtain that $g(x-t)<t / \delta$ for all $0<t<\delta$. Thus

$$
\delta(x)=\sup \left\{\delta: g(x-t)<\frac{t}{\delta} \text { for all } 0<t<\delta\right\}
$$

defines a gauge on $\mathbb{R} \backslash N$. Then $x-\delta(x)<y<x$ implies that $g(y)<$ $(x-y) / \delta(x)$. We apply Proposition 1 to obtain a right scattered set $S$ so that if $y \notin S$, then some sequence $x_{i} \searrow y$ can be found with $\delta\left(x_{i}\right) \geq \epsilon>0$. Hence for such a $y, g(y)<\left(x_{i}-y\right) / \epsilon$. It follows that, except for $y$ in a right scattered set, $g(y)=0$.

Left scattered, scattered and splattered versions of the lemma are, of course, easily formulated and just as easily proved.

The next theorem is from [5] where the scattered part of the proof is obtained by a game argument similar to Proposition 1. Later Humke and Laczkovich [8], in this Exchange, provided a category proof of this same result. Note that in the statement here the condition (3) is redundant and can be deduced from assertions (1) and (2); see [5] or [9] for this.

Theorem 5 Let $\mathcal{C}$ be a collection of closed intervals with the following properties:

(1) if $[a, b],[b, c] \in \mathcal{C}$, then $[a, c] \in \mathcal{C}$.

(2) for each $x$ there is a $\delta(x)>0$ so that $[x-t, x+t] \in \mathcal{C}$ for all $0<t<\delta(x)$.

(3) there is a countable set $N$ so that if $a, b \notin N$ then $[a, b] \in \mathcal{C}$.

Then there is a right scattered set $S_{r}$ and a left scattered set $S_{\ell}$ so that if $x<y, x \notin S_{r}$ and $y \notin S_{\ell}$, then $[x, y] \in \mathcal{C}$.

Proof. Let $Z=\left\{\frac{1}{2}(x+y): x, y \in N\right\}$. Restrict the domain of the gauge $\delta$ to exclude all points in $Z$. Let $S_{r}$ and $S_{\ell}$ be the right and left scattered sets from Proposition 1. Suppose that $x<y$ with $x \notin S_{r}$ and $y \notin S_{\ell}$. We shall find points $x^{\prime}, y^{\prime}$ not in the set $N$ such that $x \leq x^{\prime}<y^{\prime} \leq y$ and such that $x=x^{\prime}$ or $\left[x, x^{\prime}\right] \in \mathcal{C}$ and $y=y^{\prime}$ or $\left[y^{\prime}, y\right] \in \mathcal{C}$. ¿From the existence of these points we see that (1) and (3) imply that $[x, y] \in \mathcal{C}$ and the theorem is proved.

If $x \notin N$ let $x^{\prime}=x$. Otherwise, since $x \notin S_{r}$ we can find $w \notin Z$ such that $w-\delta(w)<x<w<2 w-x<\frac{1}{2}(x+y)$. Let then $x^{\prime}=2 w-x$. Since $w \notin Z$ 
and $x \in N$ we get that $x^{\prime} \notin N$. Also (2) implies that $\left[x, x^{\prime}\right] \in \mathcal{C}$. The choice for $y^{\prime}$ is similar.

The remaining theorems arise from considerations involving symmetric derivatives of real functions. If, at every point $x$,

$$
0<\liminf _{t \rightarrow 0} \frac{f(x+t)-f(x-t)}{2 t} \leq \limsup _{t \rightarrow 0} \frac{f(x+t)-f(x-t)}{2 t}<+\infty
$$

then it may be proved that $f$ is increasing off a scattered set. One shows that such a function is increasing on the set of its points of continuity (eg. [11, p. 184]) and then invokes a theorem of Charzyński [2] asserting that a function with this property is continuous off a scattered set. The article [5] contains another approach. Here we show that once the countable exceptional set is granted (see [5] or [9] for an elementary proof) Proposition 1 easily gives the rest of the proof.

Theorem 6 Let $f$ be a real function and $N$ a countable set. Suppose that $f$ is [strictly] monotone on $\mathbb{R} \backslash N$ and that

$$
-\infty<\liminf _{t \rightarrow 0} \frac{f(x+t)-f(x-t)}{2 t} \leq \limsup _{t \rightarrow 0} \frac{f(x+t)-f(x-t)}{2 t}<+\infty
$$

at every point $x \in \mathbb{R} \backslash N$. Then $f$ is [strictly] monotone off a scattered set.

Proof. Let us just show the nondecreasing version. Let $Z$ denote the set of points of the form $(x+y) / 2$ for $x, y \in N$. For each $x \in \mathbb{R} \backslash Z$ we use the following natural gauge

$$
\delta(x)=\sup \left\{\delta:\left|\frac{f(x+h)-f(x-h)}{2 h}\right|<\frac{1}{\delta} \text { for all } 0<h<\delta \leq 1\right\} .
$$

If $x-\delta(x)<y<x$, then $|f(2 x-y)-f(y)|<2(x-y) / \delta(x)$. By Proposition 1 , except for $y$ in a right scattered set $S_{r}$ there is a sequence $x_{i} \searrow y$ with $\delta\left(x_{i}\right)$ bounded away from zero. Consequently $f\left(2 x_{i}-y\right) \rightarrow f(y)$. If, in addition, $y \in N$, then $2 x_{i}-y$ cannot be in $N$ since each point $x_{i}$ is not in the set $Z$. Hence, for $y \in N \backslash S_{r}$, we have

$$
f(y)=\lim _{x \rightarrow y+, x \notin N} f(x) .
$$

A dual argument would give a left scattered set $S_{\ell}$ such that, for $y \in N \backslash S_{\ell}$,

$$
f(y)=\lim _{x \rightarrow y-, x \notin N} f(x) .
$$


Hence $f$ cannot decrease on the set

$$
(\mathbb{R} \backslash N) \cup\left(N \backslash S_{r}\right) \cup\left(N \backslash S_{\ell}\right)=\left(\mathbb{R} \backslash\left(S_{r} \cap S_{\ell}\right)\right)
$$

without already decreasing on $(\mathbb{R} \backslash N)$.

If in the previous theorem we know only that

$$
\limsup _{t \rightarrow 0} \frac{f(x+t)-f(x-t)}{2 t}<+\infty
$$

in place of (3), then the limit in (4) would become $f(y) \geq \lim _{x \rightarrow y+, x \notin N} f(x)$.for $y \in N \backslash S_{r}$ and the limit in (5) would become $f(y) \leq \lim _{x \rightarrow y-}, x \notin N f(x)$. and $f\left(y_{1}\right)>f\left(y_{2}\right)$ it must be the case that $y_{1} \in S_{\ell}$ or $y_{2} \in S_{r}$. We therefore have the following theorem (see [5] for a closely related monotonicity theorem).

Theorem 7 Let $f$ be a real function and $N$ a countable set. Suppose that $f$ is increasing [nondecreasing] on $\mathbb{R} \backslash N$ and that

$$
\limsup _{t \rightarrow 0} \frac{f(x+t)-f(x-t)}{2 t}<+\infty
$$

at every point $x \in \mathbb{R} \backslash N$. Then there is a right scattered set $S_{r}$ and a left scattered set $S_{\ell}$ so that if $x<y, x \notin S_{\ell}$ and $y \notin S_{r}$, then $f(x)<f(y)$ $[f(x) \leq f(y)]$. In particular $f$ is increasing [nondecreasing] off a splattered set.

Before leaving this discussion of Theorem 6 we should indicate that it provides another proof of a well-known theorem of Charzyński [2]. Sierpiński derivates is continuous except on a countable set. Charzyński showed that the exceptional set is scattered. Using the proof of Theorem 6 and assuming that the set of discontinuity points is in fact countable we get quickly that this set is scattered. Charzyński's original proof uses an argument based on Lemma 4.

Evans and Larson [4] obtained a closely related theorem: if $f$ is measurable and $f(x+h)+f(x-h)-2 f(x)=O(h)($ as $h \rightarrow 0)$ everywhere, then again the set of discontinuities of $f$ is scattered. Our methods will prove this too once the countability of the set of discontinuities is established. For a more detailed account of Charzyński's theorem and the theorem of Evans and Larson

\section{References}

[1] G. Cantor. Über unendliche, lineare Punktmannigfaltigkeiten, Math. Ann., 23:453-488, 1884. 
[2] Z. Charzyński. Sur les fonctions dont la derivée symetrique est partout finie. Fund. Math., 21:214-225, 1931.

[3] R. O. Davies and F. Galvin. Solution to query 5. Real Anal. Exchange, 2:74-75, 1976.

[4] M. J. Evans and L. Larson. The continuity of symmetric and smooth functions. Acta. Math. Hungar., 43:251-257, 1984.

[5] C. Freiling. Symmetric derivates, scattered and semi-scattered sets. Trans. American Math. Soc., 318:705-720, 1990.

[6] C. Freiling and B. S. Thomson. Scattered Sets, Chains and the Baire Category Theorem. Real Anal. Exchange, (this issue).

[7] E. W. Hobson. The theory of functions of a real variable (2nd ed.) I,II, Cambridge (1926).

[8] P. D. Humke and M. Laczkovich. An elementary proof of Freiling's symmetric covering lemma. Real Anal. Exchange, 16:549-551, 1990/91.

[9] D. Preiss and B. S. Thomson. A symmetric covering theorem. Real Anal. Exchange, 14 no. 1:253-254, 1988/89.

[10] T. Viola. Sur la détermination d'une fonction discontinue par sa dérivée unilatérale. Ann. École Norm., (3) 50:72-125, 1933.

[11] B. S. Thomson. Symmetric Properties of Real Functions. Monographs and Textbooks in Pure and Appl. Math. \#183, Marcel Dekker Inc., 1994. 